Original Article - Clinical Science

\title{
Differences in practice of ophthalmology by gender in Australia
}

\author{
Tiffany CS Lo MBBS BMedSci, ${ }^{1,2}$ Sophie L Rogers Mepi, ${ }^{2}$ Anthony J Hall MBBS MD ${ }^{3}$ \\ and Lyndell L Lim FRANZCO DMedSci ${ }^{1,2}$
}

1. The Royal Victorian Eye \& Ear Hospital, East Melbourne VIC 3002, Australia

2. The Centre for Eye Research Australia (CERA), East Melbourne VIC 3002, Australia

3. Department of Ophthalmology, Alfred Health, Melbourne VIC 3004, Australia

Correspondence: A/Prof Lyndell Lim, Centre for Eye Research Australia, Royal Victorian Eye and Ear Hospital, Peter Howson Wing, Level 7, 32 Gisborne Street, East Melbourne 3002 VIC, Australia

Email: limllp@unimelb.edu.au

Short running title: Differences in practice by gender Received 18 December 2018; accepted 21 April 2019 Funding sources / Financial disclosure: Lyndell L Lim (GNT 1109330) is supported by a National Health and Medical Research Council (NHMRC) Early Career Fellowship. CERA receives funding from the Australian Government.

Conflict of interest: None

Keywords: Clinical practice, gender, ophthalmologist, workforce, remuneration

This is the author manuscript accepted for publication and has undergone full peer review but has not been through the copyediting, typesetting, pagination and proofreading process, which may lead to differences between this version and the Version of Record. Please cite this article as doi: 10.1111/ceo.13523

This article is protected by copyright. All rights reserved. 


\section{ABSTRACT}

Importance: The number of females practising ophthalmology is rising. It is known that practice patterns between female and male ophthalmologists differ. Understanding the differences will help to inform future workforce planning.

Background: To investigate the differences in clinical practice between female and male ophthalmologists in Australia.

Design: Cross-sectional study.

Participants: Ophthalmologists participating in the Royal Australian \& New Zealand College of Ophthalmologists workforce survey, and/or Medicine in Australia:

Balancing Employment \& Life survey, and those who made claims from Medicare Benefits Schedule Australia.

Methods: Combined analysis of de-identified 2014 data from the surveys and Medicare Benefits Schedule.

Main outcome measures: Hours worked, service provision, remuneration and social circumstances.

Results: Female ophthalmologists provided 35\% fewer services per ophthalmologist per year ( 2834 vs 4328 ) than male ophthalmologists. Female ophthalmologists received approximately half the annual income of male ophthalmologists; median self-reported net personal annual income was AUD122500 (IQR 96000-225000) for females compared to AUD245000 (IQR180000-365000) for males $(p=0.01)$. The median self-reported hours worked per week was 35.0 (IQR 28.0-46.0) for females and 41.8 (IQR 36.5-48.5) for males ( $p=0.04$ ). A higher proportion of females practise in medical subspecialties while a higher proportion of males practise in surgical subspecialties.

Conclusions and relevance: Female ophthalmologists earn less compared to male ophthalmologists after accounting for lower service provision and hours worked. Difference in income may be partially accounted for by higher total number of 
services and procedural services provided by male ophthalmologists. Understanding differences between female and male ophthalmologists will help to inform future medical workforce planning.

\section{INTRODUCTION}

Due to the increasing number of women entering medicine and ophthalmology there has been increasing interest in differences in the practice profile of female and male ophthalmologists. Currently, $52.1 \%$ of Australian medical students are female, and $42.7 \%$ of all registered medical practitioners in Australia are female. ${ }^{1,2}$ In 2017, $21.2 \%$ of fellows and $35.1 \%$ of ophthalmology trainees were female in the Royal Australian \& New Zealand College of Ophthalmologists (RANZCO). ${ }^{3}$ Studies around the world have found that female doctors earn less than their male counterparts, even after accounting for number of hours worked ${ }^{4-8}$, and female ophthalmologists report doing less procedural work than their male counterparts. ${ }^{9,10}$

Few studies have been conducted investigating the differences in practice patterns, social circumstances and remuneration between female and male ophthalmologists in Australia.

A survey of RANZCO fellows in 2005 conducted by Danesh-Meyer et al ${ }^{5}$ found that a higher proportion of female ophthalmologists worked fewer hours - and after adjusting for hours worked, their average annual income was still less than that of males. Female ophthalmologists were also less likely than males to have children, yet they were more likely to contribute more to child-rearing activity. ${ }^{5}$ This discrepancy was further substantiated by the finding that in the 2014/2015 financial year, male ophthalmologists had an average taxable income $58 \%$ higher than that of their female counterparts. ${ }^{11}$ 
This study aims to determine the differences in practice patterns and social circumstances of Australian ophthalmologists by gender of clinician to inform ophthalmology workforce planning.

\section{METHODS}

This is a cross-sectional study. Ethics Approval for the study was granted on 13 January 2017 by the RANZCO Human Research Ethics Committee Project number 68.16. No identified data has been used in this study. Data was obtained from deidentified reports that are not able to be re-identified.

Analysis was conducted on existing de-identified 2014 data from the following sources:

1) The Medical Board of Australia

The public national register of medical practitioners is published online every 3 months. As it is a legal requirement for all medical practitioners to be registered, this provides accurate reports of Australia's medical workforce profile. The medical practitioner registrant data reports include statistical breakdown of registrants by specialty, age and gender.

2) Medicare Australia

Medicare billing data from Medicare Benefits Schedule (MBS) according to gender and age of provider for the most common ophthalmology services; initial consultation, subsequent consultation, paediatric consultation, cataract surgery, intraocular injection and retinal laser. Medicare funds, at least in part, the vast majority of non-cosmetic ophthalmology consultations and procedures in Australia. 
3) Medicine in Australia: Balancing Employment and Life (MABEL) study ${ }^{\mathbf{1 2}}$ Longitudinal survey of Australian medical practitioners focusing on work-life balance issues in doctors. Practicing clinicians are identified through the Australasian Medical Publishing Company's Medical Directory which is a national database of doctors for mailing purposes. Invitations are sent by mail or email for doctors to complete an electronic or paper copy of the MABEL questionnaire.

4) RANZCO online anonymous survey on ophthalmologists' work patterns Survey sent out to RANZCO members with questions including location of practice (metropolitan, regional or rural), type of practice (solo or group) and subspecialisation.

Age was categorised into five groups; $<35$ years, $35-44$ years, $45-54$ years, $55-$ 64 years and 65 years and older.

\subsection{Data analysis}

All data were imported into Stata/IC for Windows version 10.0 (Statacorp, College Station, Texas, USA) and Microsoft Excel version 15.27 for analysis. Graphs were made in Stata/IC for Windows version 14.2. Mean and standard deviation (SD) were reported for normally-distributed data, and median and interquartile ranges (IQR) for non-parametric data. Fisher exact tests were performed on binary and categorical variables and Wilcoxon rank-sum tests on non-parametric continuous variables. P-values $<0.05$ were considered statistically significant.

\section{RESULTS}

\subsection{Demographics}


A total of 954 Australian ophthalmology providers claimed from MBS in 2014, 843 $(88.4 \%)$ responded to the RANZCO survey. Of the 920 ophthalmologists invited to complete the MABEL survey 91 responded. There were 27 female and 64 male respondents to the MABEL survey, and 187 female and 656 male respondents to the RANZCO workforce survey. A higher proportion of younger females were represented by all three sources of data, while for males there was more even distribution among age groups (Figure 1).

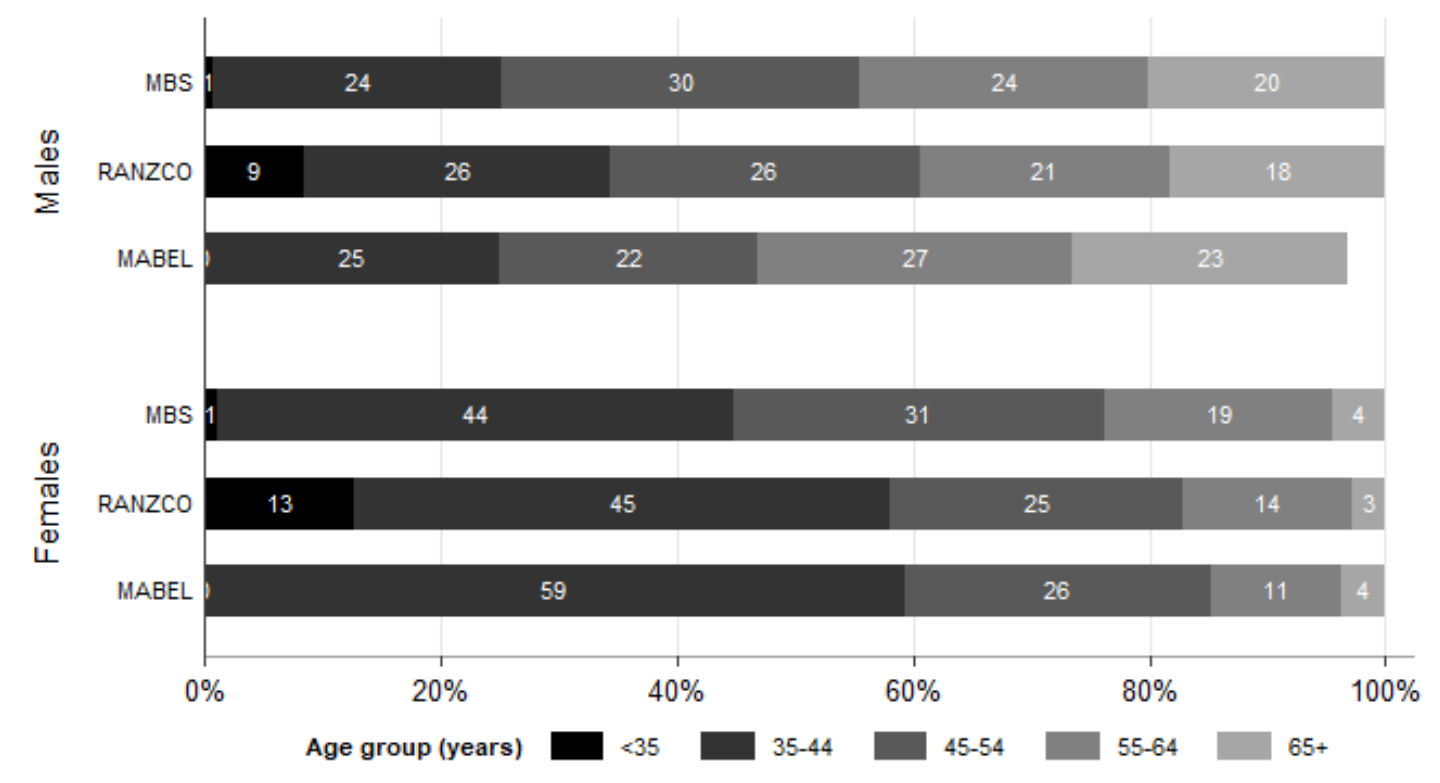

Figure 1: Demographics of ophthalmologists from data sources

\subsection{Subspecialisation}

From the results of the RANZCO survey, a higher proportion of females practise in medical subspecialties (e.g. medical retina, uveitis or neuro-ophthalmology), while a higher proportion of males practice in surgical subspecialties (e.g. surgical retina and cornea) (Figure 2). Subspecialty data and disaggregation are not available from or collected by the MABEL survey or MBS. 


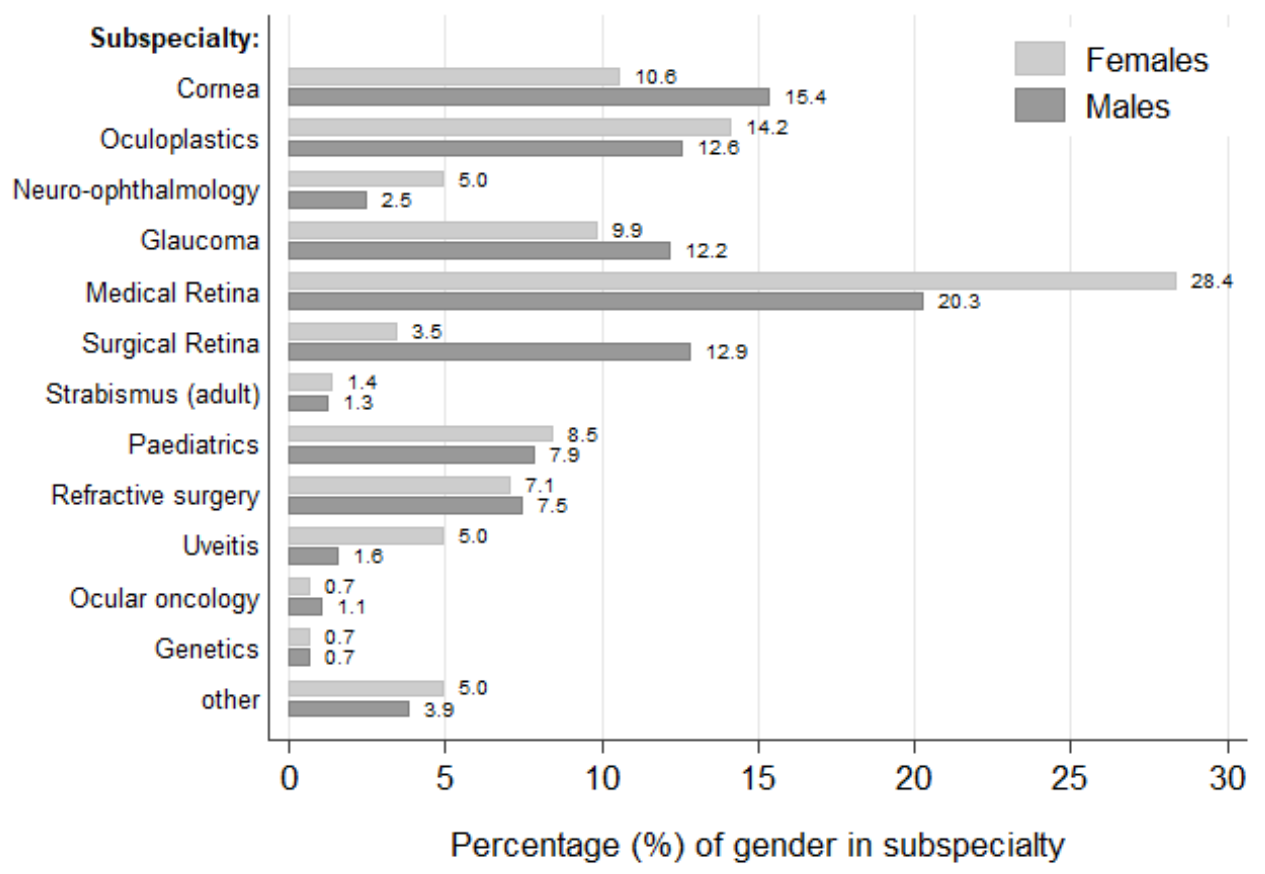

Figure 2: Ophthalmology subspecialisation by gender (RANZCO survey)

\subsection{Location and hours of work}

A lower proportion of female RANZCO survey respondents provided services at regional or rural public facilities compared to males: $18.4 \%$ (29/158) compared to $27.3 \%(153 / 560 ; p=0.02)$. Similarly, fewer females had a regional or rural practice: $18.8 \%(30 / 160)$ compared to $40.1 \%(224 / 558 ; p<0.001)$. Only $13 \%(26 / 187)$ female RANZCO survey respondents own and operate a solo practice compared to $26.5 \%(174 / 656)$ of male respondents $(p=0.003)$. However, there was no difference in the proportion who reported being an associate in a group practice: $52.9 \%(99 / 187)$ females compared to $50.6 \%(332 / 656)$ males $(p=0.69)$.

Female MABEL survey respondents reported a median of 35.0 (IQR 28.0-46.0) hours worked per week while male respondents reported a median of 41.8 (IQR 36.5-48.5) 
hours worked per week $(p=0.04)$. Half of all female ophthalmologists gave an initial consultation duration between 20-30 minutes whilst half the males gave initial consultations of 15-25 minutes duration (females median: 20 minutes [IQR 20-30]; males median: 20 minutes [IQR 15-25]; Wilcoxon rank-sum test, $\mathrm{p}=0.01$ ).

\subsection{Remuneration}

Female respondents reported median net annual income of AUD122500 (IQR 96000225000) while male MABEL respondents reported median net annual income of AUD245000 (IQR 180000-365000) $(p=0.01)$.

Overall, females claimed for fewer MBS services compared to males, especially in the younger age groups. Females provided $35 \%$ fewer services per ophthalmologist per year (2834/female ophthalmologist vs 4328/male ophthalmologist), although females aged $35-65$ years provided more paediatric consultations per clinician compared with males in the same age group. Fees per service were similar between males and females (Figure 3). 

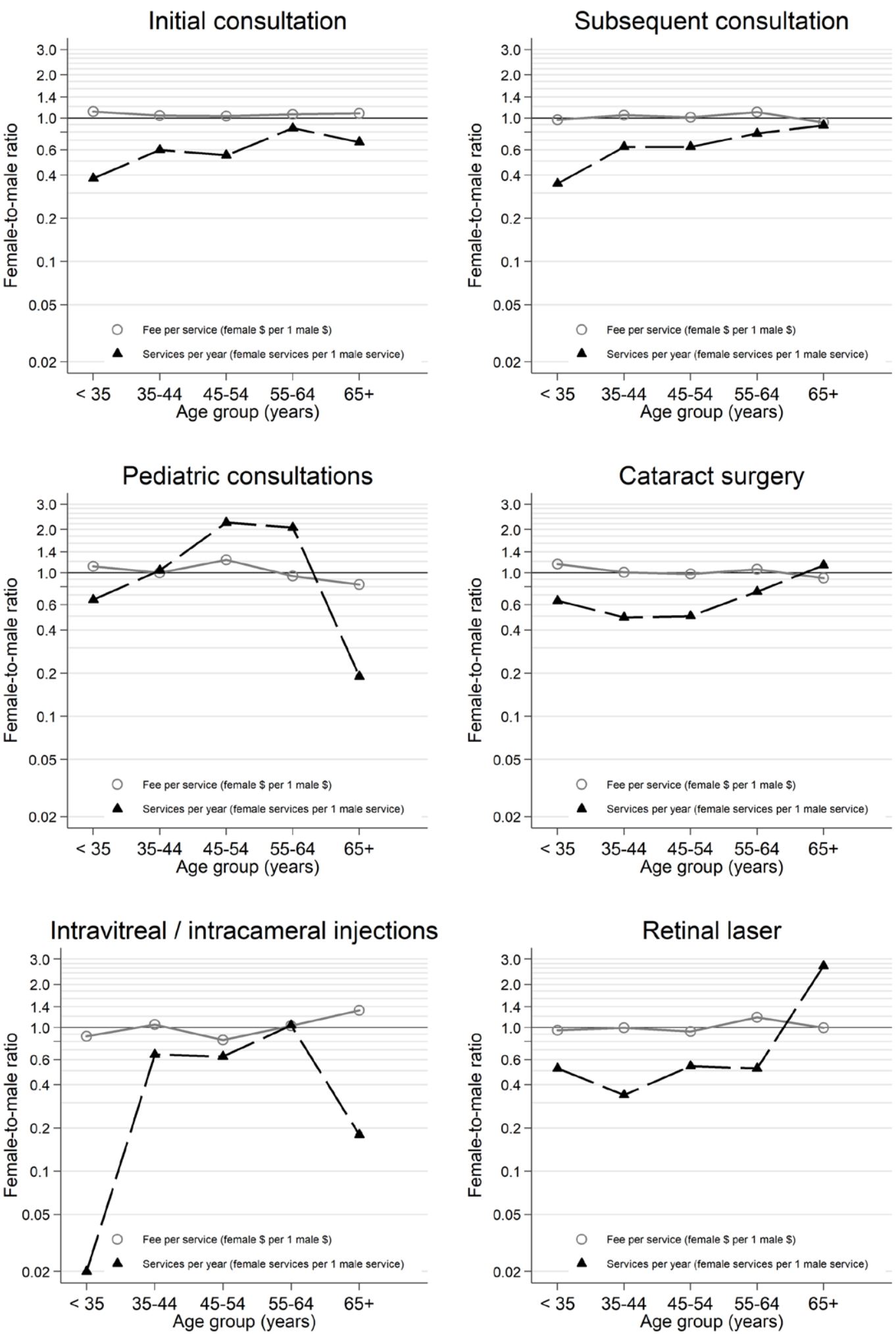

This article is protected by copyright. All rights reserved. 
Figure 3: Female-to-male ratios of number of services per provider and fees per service (MBS data). Grey circles indicate the fee charged per service by a female doctor relative to every $\$ 1$ charged by a male doctor. Black triangles indicate the average number of services per provider performed each year by female doctors relative to every one service per male doctor. A value of 1.0 (shown by the black horizontal line) means parity in charging or annual service provision rate between female and male ophthalmologists. A data point of greater than 1.0 means that an excess in fees or number of services performed by female compared to male ophthalmologists. A value below 1.0 indicates where female ophthalmologists are charging less, or performing fewer services per provider than their male colleagues.

\subsection{Career satisfaction}

There was no significant difference in career satisfaction among female and male MABEL survey respondents. The majority of females and males were satisfied overall $(26 / 27$ vs $59 / 63$, respectively; $p=1.0)$, satisfied with hours of work (24/27 vs $49 / 63$, respectively; $p=0.26$ ), and satisfied with remuneration $21 / 27$ females versus $57 / 63$ males, $p=0.17$ ).

\subsection{Marital status \& family situation}


There was no significant difference between the proportion of females and males with a partner/spouse $(24 / 27$ vs $59 / 64, p=0.69)$ in the MABEL survey. However, spouses of female ophthalmologists were more likely to be in employment compared with spouses of male ophthalmologists ( $22 / 24$ vs $34 / 59, p=0.004)$. The majority $(15 / 24)$ of spouses of female ophthalmologists were in full-time employment (Figure 4).

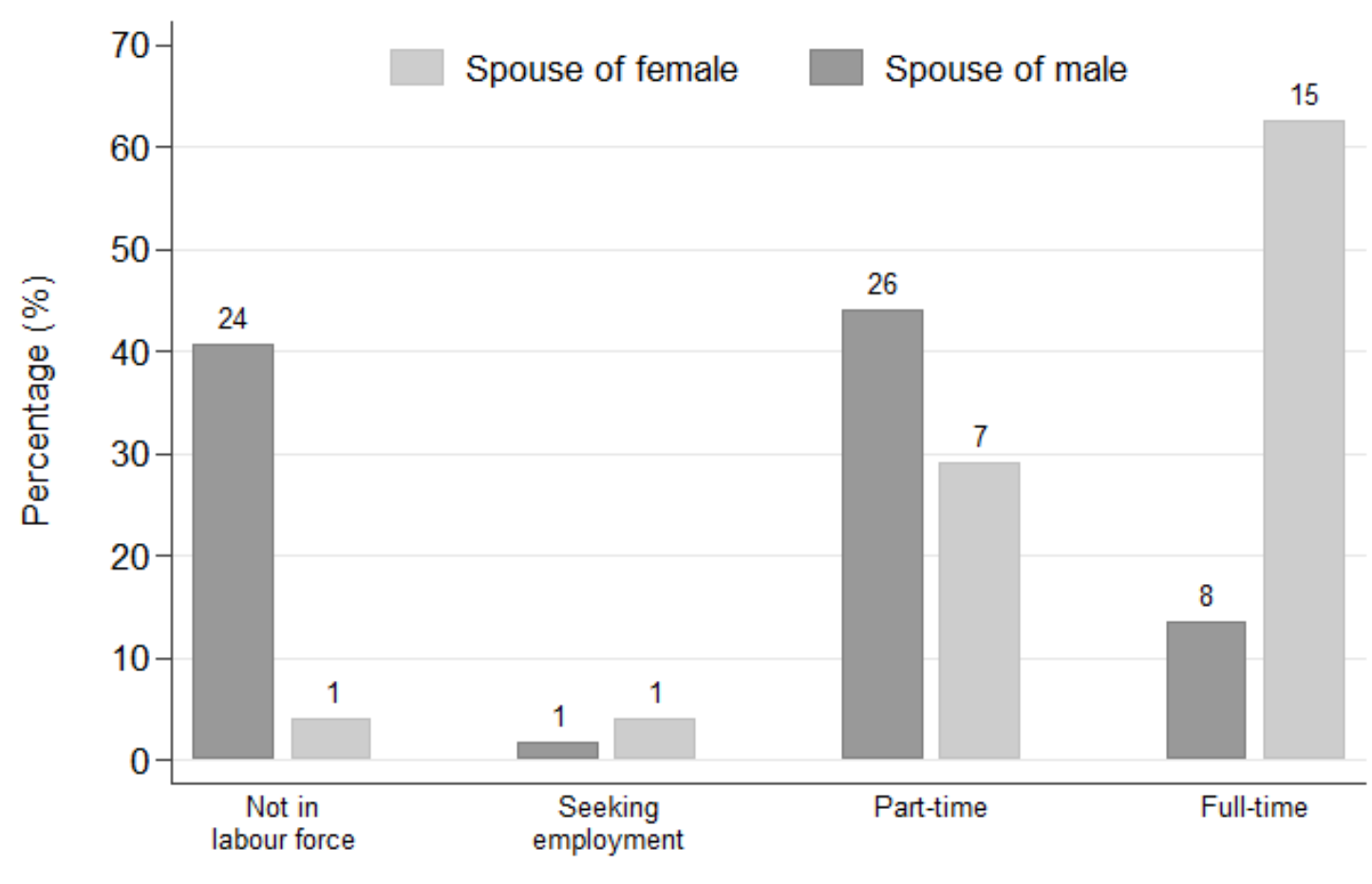

Note: Numbers on bars indicate the count of respondents per category

Figure 4: Employment status of spouse (MABEL survey)

Female and male MABEL respondents were similarly likely to have children (20/27 vs $41 / 64, p=0.47)$

\section{DISCUSSION}


This study revealed differences between female and male ophthalmologists in terms of remuneration, subspecialisation, location and hours of work, and spouse employment status. In this study, females worked approximately $16 \%$ fewer hours than males, while their median net annual income was almost 50\% less than males. Females were more likely to be practising in medical subspecialties, while males were more likely to be practising in procedural subspecialties. Males were also more likely to own a solo practice, work in rural or regional centres and have a spouse who was not in the labour force.

Our results demonstrate that female ophthalmologists work fewer hours and provide fewer services. However, this needs to be interpreted in the context that females had longer consults. Thus, the reduction in services is not just accounted for by the fewer hours worked and demonstrates an increased time spent on each service. In other studies ${ }^{13,14}$ an increase in duration of service has been associated with greater patient satisfaction and lower malpractice claims. Our study, however, did not attempt to determine if that is the case in ophthalmology in Australia In addition, the previous study on RANZCO fellows conducted by Danesh-Meyer et al ${ }^{5}$ showed that while females work fewer hours compared to men, they are still working a significant number of hours (over half work 30 hours or more per week) while taking on more family commitments. Jinapriya et $\mathrm{al}^{9}$ found that while female ophthalmologists worked similar hours to male ophthalmologists, they contribute more to child-caring activities. In keeping with the study by Danesh-Meyer et $\mathrm{al}^{5}$, we also found that females earn less than their male counterparts even when accounting for number of hours worked. Weeks \& Wallace ${ }^{15-24}$ analysed surveys conducted between 19922001 by the American Medical Association comparing annual income of specialists by gender. They found that female doctors, including ophthalmologists, general surgeons, general physicians, emergency physicians and anaesthetists, received 
lower annual incomes than their male counterparts, after accounting for number of working hours. ${ }^{15-24}$ Our findings also echo recent Medicare data from the United States, where American female ophthalmologists earn less and made fewer clinical billings than their male counterparts. ${ }^{7}$

In this study, since we found that fees per service were similar between females and males, this disparity in income may be due to our findings of the longer duration of time females spent on consultations. We also found that females provided a higher number of paediatric consultations per clinician, which tend to be longer in duration than adult consultations. The relative lower remuneration in paediatric ophthalmology may be responsible for the identified relative workforce shortage in paediatric ophthalmology. It may also be a disincentive for male ophthalmologists choosing the subspecialty as we found male ophthalmologists were more likely to be the sole or main income earner for the family, as opposed to female ophthalmologists who were more likely to have a spouse in full-time employment.

Our findings of the larger representation of females in medical, rather than surgical subspecialties may be another contributing factor in income disparity. We found a higher proportion of females were working in medical subspecialties, such as medical retina and uveitis, while there was more male representation in surgical subspecialties, such as surgical retina and cornea. As a result, it is not surprising that we found females claimed fewer procedural services per clinician compared to males, and therefore lower remuneration. This is in keeping with findings of a survey of Canadian ophthalmologists ${ }^{9}$, where male respondents were found to have a higher procedural workload. Fewer opportunities for surgical exposure during training may be a factor in fewer females choosing a career in a surgical subspecialty. Gibson et $\mathrm{al}^{25}$ conducted a survey among ophthalmology trainees in the UK and found that female trainees had fewer wet lab sessions and completed 
fewer cataract surgeries compared with male trainees. In our study, a higher proportion of males also owned and operated a solo practice, which other studies suggest increases income. ${ }^{4,21}$

In concordance with findings of previous surveys in Australia, New Zealand and Canada $^{5,9,10}$ females were more likely to have a partner in full-time employment while males were more likely to have a partner not in the labour force. This may be an explanation for the higher proportion of males involved in rural or regional practice. Without the need to find employment for a spouse, male ophthalmologists may be more readily able to work in a rural or regional setting. Conversely, the fact that male ophthalmologists are more likely to work in rural or regional practice may negatively impact on their partners' opportunities to seek employment.

The survey component of the data compiled for this study has inherent limitations due to its subjective nature, voluntary participation, recall bias and survey response rates. In particular, there was a low response rate of $10 \%$ in the MABEL survey which limits the generalizability of the survey data. Consequently, the results may not reflect practice patterns and social circumstances of all ophthalmologists. In the absence of data on variance, survey results should be interpreted as descriptive and statistical comparisons should be interpreted with caution. The strength of this study lies in analysis of objective remuneration data. The data completely captures income from the most common services provided by ophthalmologists. However, it is recognized that the study cannot account for other sources of income (e.g. nonclinical work).

Remuneration, subspecialisation, duration of consultation, location and hours of work, and spouse employment status are all contributing factors to the difference in practice patterns of female and male ophthalmologists. 
Further studies are required to investigate determinants of differences in clinical practice between female and male ophthalmologists to inform future workforce planning. Suggested avenues for further research that can lead to changes in workforce disparities include investigation into the barriers to women entering more procedural subspecialties, and barriers to men entering medical subspecialties. Further investigation into the number of surgical procedures per female or male ophthalmologist in the surgical subspecialties may also shed some light onto whether females are less likely to recommend surgical interventions than their male counterparts, as suggested by Wilson et al's study ${ }^{26}$ among general surgeons in the US. However, as this line of investigation requires individual, potentially identifying data, this was not an avenue of research that we could pursue. Although we found similar career satisfaction among female and male ophthalmologists, Danesh-Meyer et $\mathrm{al}^{5}$ found that females experienced greater frustration with balancing work and family commitments, and Jinapriya et al $^{9}$ found that whilst women worked similar hours, they contribute significantly more to caring for children. Yet the issue of work-life balance is not unique to women. There has been a trend amongst younger generations of doctors, both female and male, towards opting for careers with better work-life balance. Dorsey et $\mathrm{al}^{27}$ found that the proportion of female medical students choosing specialties with a controllable lifestyle rose from $18 \%$ in 1996 to $36 \%$ in 2003 , while the proportion of men choosing specialties with a controllable lifestyle was higher, rising from $28 \%$ to $45 \%$ over the same time period. Research investigating factors affecting career choices among ophthalmology trainees in Australia may help guide workforce planning for the future.

\section{Acknowledgements}


We would like to thank RANZCO (The Royal Australian \& New Zealand College of Ophthalmologists) and MABEL (Medicine in Australia: Balancing Employment and Life Survey) for providing their workforce survey data.

The study used data from the MABEL longitudinal survey of doctors conducted by the University of Melbourne and Monash University. The MABEL research team bears no responsibility for how the data has been analysed, used or summarised in this study. Funding for MABEL comes from the NHMRC (Health Services Research Grant: 2008-2011; and Centre of Research Excellence in Medical Workforce Dynamics: 2012-2017) with additional support from the Department of Health (in 2008) and Health Workforce Australia (in 2013). 


\section{REFERENCES}

1. Annual tables: Medical Deans Australia and New Zealand; 2017. http://www.medicaldeans.org.au/statistics/annualtables/. (Accessed Nov 2016)

2. Medical Board of Australia registrant data: Medical Board of Australia; 2018. http://www.medicalboard.gov.au/News/Statistics.aspx. (Accessed July 2018) 3. RANZCO. RANZCO 2016 - 2017 Annual Report New South Wales, Australia 2017. https://ranzco.edu/ArticleDocuments/227/Annual Report 2016.17.pdf.aspx?Embed $=Y$. (Accessed March 2019)

4. Baker LC. Differences in earnings between male and female physicians. New England Journal of Medicine. 1996;334(15):960-4.

5. Danesh-Meyer HV, Deva NC, Ku JY, et al. Differences in practice and personal profiles between male and female ophthalmologists. Clinical \& Experimental Ophthalmology. 2005;35(4):318-23.

6. Desai $T$, Ali $S$, Fang $X$, et al. Equal work for unequal pay: the gender reimbursement gap for healthcare providers in the United States. Postgraduate Medical Journal. 2016;92(1092):571-5.

7. Reddy AK, Bounds GW, Bakri SJ, et al. Differences in Clinical Activity and Medicare Payments for Female vs Male Ophthalmologists. JAMA ophthalmology. 2017;135(3):205-13.

8. Ly DP, Seabury SA, Jena AB. Differences in incomes of physicians in the United States by race and sex: observational study. BMJ. 2016;353:i2923.

9. Jinapriya $D$, Cockerill $R$, Trope GE. Career satisfaction and surgical practice patterns among female ophthalmologists. Canadian journal of ophthalmology Journal canadien d'ophtalmologie. 2003;38(5):373-8.

10. McAlister $\mathrm{C}$, Jin $\mathrm{YP}$, Braga-Mele $\mathrm{R}$, et al. Comparison of lifestyle and practice patterns between male and female Canadian ophthalmologists. Canadian journal of ophthalmology Journal canadien d'ophtalmologie. 2014;49(3):287-90. 
11. Taxation Statistics 2014-2015 Individuals: Australian Government: Australian Taxation Office; 2017. https://data.gov.au/dataset/taxation-statistics-201415/resource/169f8d12-006c-441c-95c5-91d71fe3b9d4?inner span=True. (Accessed Apr 2018)

12. Medicine in Australia: Balancing Employment and Life: The University of Melbourne; 2018. https://melbourneinstitute.unimelb.edu.au/mabel/home - mabel. (Accessed Sept 2018)

13. Levinson W, Roter DL, Mullooly JP, et al. Physician-patient communication: the relationship with malpractice claims among primary care physicians and surgeons. JAMA. 1997;277(7):553-9.

14. Lin C-T, Albertson GA, Schilling LM, et al. Is patients' perception of time spent with the physician a determinant of ambulatory patient satisfaction? Archives of internal medicine. 2001;161(11):1437-42.

15. Weeks WB, Wallace $A$. The influence of race and gender on family physicians' annual incomes. Journal of the American Board of Family Medicine : JABFM. 2006;19(6):548-56.

16. Weeks WB, Wallace AE. Gender Differences in Diagnostic Radiologists' Annual Incomes. Academic Radiology. 2006;13(10):1266-73.

17. Weeks WB, Wallace AE. Association of Race and Gender with General Surgeons' Annual Incomes. Journal of the American College of Surgeons. 2006;203(4):558-67.

18. Weeks WB, Wallace AE. Race and gender differences in general internists' annual incomes. Journal of general internal medicine. 2006;21(11):1167-71.

19. Weeks WB, Wallace AE. The influence of provider sex on neurologists' annual incomes. Clinical Neurology and Neurosurgery. 2007;109(1):38-44.

20. Weeks WB, Wallace AE. Race and Gender Differences in Pediatricians' Annual Incomes. Ambulatory Pediatrics. 2007;7(2):196-200. 
21. Weeks WB, Wallace AE. Gender differences in ophthalmologists' annual incomes. Ophthalmology. 2007;114(9):1696-701.

22. Weeks WB, Wallace AE. Differences in the annual incomes of emergency physicians related to gender. Acad Emerg Med. 2007;14(5):434-40.

23. Weeks WB, Wallace AE. Gender differences in the annual income of psychiatrists. Psychiatric Services. 2007;58(4):515-20.

24. Weeks William B, Wallace $H$, Amy $E$, et al. Gender Differences in Anesthesiologists' Annual Incomes. Anesthesiology. 2007;106(4):806-11.

25. Gibson A, Boulton $M$, Watson $M$, et al. The first cut is the deepest: basic surgical training in ophthalmology. Eye. 2005;19(12):1264.

26. Wilson NP, Wilson FP, Neuman M, et al. Determinants of surgical decision making: a national survey. The American Journal of Surgery. 2013;206(6):970-8.

27. Dorsey ER, Jarjoura $D$, Rutecki GW. The influence of controllable lifestyle and sex on the specialty choices of graduating US medical students, 1996-2003. Academic Medicine. 2005;80(9):791-6. 


\section{University Library}

\section{- M M N E R VA A gateway to Melbourne's research publications}

Minerva Access is the Institutional Repository of The University of Melbourne

Author/s:

Lo, TCS;Rogers, SL;Hall, AJ;Lim, LL

Title:

Differences in practice of ophthalmology by gender in Australia

Date:

2019-09-01

Citation:

Lo, T. C. S., Rogers, S. L., Hall, A. J. \& Lim, L. L. (2019). Differences in practice of ophthalmology by gender in Australia. CLINICAL AND EXPERIMENTAL OPHTHALMOLOGY, 47 (7), pp.840-846. https://doi.org/10.1111/ceo.13523.

Persistent Link:

http://hdl.handle.net/11343/285869 